\title{
Thrombotic Microangiopathy Following Chemotherapy with S-1 and Cisplatin in a Patient with Gastric Cancer: A Case Report
}

\author{
JUN MUTO ${ }^{1}$, HIROSHI KISHIMOTO ${ }^{2}$, YASUO KAIZUKA ${ }^{3}$, MITSURU KINJO ${ }^{4}$, \\ HIDEFUMI HIGASHI ${ }^{1}$ and FUMIAKI KISHIHARA ${ }^{1}$ \\ Departments of ${ }^{1}$ Surgery, ${ }^{2}$ Nephrology and ${ }^{4}$ Pathology, Steel Memorial Yawata Hospital, Kitakyushu, Japan; \\ ${ }^{3}$ Intensive Care Unit, Steel Memorial Yawata Hospital, Kitakyushu, Japan
}

\begin{abstract}
Background: Thrombotic microangiopathy (TMA) represents a spectrum of serious disorders characterized by occlusive microvascular thrombosis, thrombocytopenia and end-organ damage. TMA is associated with a broad range of conditions and is also a well-described complication of both cancer and its treatment. Case Report: A 77-year-old Japanese woman underwent $S-1$ and cisplatin chemotherapy for treatment of advanced gastric cancer with multiple lymph node and liver metastases. She was found with severe anemia and thrombocytopenia during the third course of chemotherapy. She was diagnosed with TMA based on thrombocytopenia, schistocytosis, hemolytic anemia and renal dysfunction. She underwent treatment with plasmapheresis; however, her response to treatment was poor and died on day 16 of hospitalization. The autopsy performed revealed microthrombi in the glomeruli and tubulonecrosis in the kidneys. Conclusion: This is the first case report of TMA in association with the use of $S-1$ and cisplatin. Therapists have to take account of TMA when using $S-1$ and cisplatin treatment.
\end{abstract}

Thrombotic microangiopathy (TMA) represents a spectrum of serious disorders characterized by occlusive microvascular thrombosis, thrombocytopenia and end-organ damage. TMA

This article is freely accessible online.

Abbreviations: TMA, Thrombotic microangiopathy; TTP, thrombotic thrombocytic purpura; ADAMTS13, a disintegrin and metalloprotease with thrombospondin type 1 motif, member 13; HER2, human epidermal growth factor receptor 2.

Correspondence to: Jun Muto, Department of Surgery, Steel Memorial Yawata Hospital, 1-1-1 Harunomachi, Yawata higashi-ku, Kitakyushu 805-8508, Japan. Tel: +81 936723176, Fax: +81 936719445, e-mail: muto@surg2.med.kyushu-u.ac.jp

Key Words: Thrombotic microangiopathy, thrombotic thrombocytic purpura, S-1, cisplatin. ranges from typical thrombotic thrombocytopenic purpura (TTP) to localized renal TMA $(1,2)$. This disease is associated with a broad range of conditions, including infection, organ transplantation, autoimmune disease and pregnancy (3). TMA is also a well-described complication of both cancer and its treatment (4). Establishment of a causal relationship between a specific chemotherapeutic agent and TMA is difficult because the malignancy itself can induce TMA. Multiple chemotherapeutic agents are often used for a single patient, making it difficult to determine the most effective drug $(5,6)$.

Diagnosis of TTP, one of the typical phenotypes of TMA, is based on the presence of five specific signs and symptoms: thrombocytopenia, microangiopathic hemolytic anemia, neurological abnormalities, renal failure and fever (2). TTP results from deficiency in the activity of a disintegrin and metalloprotease with thrombospondin type 1 motif, member 13 (ADAMTS13). Deficiency in ADAMTS13 activity leads to accumulation of ultra-large von Willebrand factor multimers and subsequent uncontrolled platelet thrombus formation in the microvasculature $(7,8)$. ADAMTS13 activity of $<20 \%$ is used as a criteria of diagnosis of TTP (1). Plasmapheresis is the key treatment that has decreased the mortality rate from $85 \%-95 \%$ to $10 \%-20 \%$ (1). Treatment with corticosteroids and recombinant human soluble thrombomodulin has also been reported (2).

We herein report a case of severe TMA that occurred in a patient with gastric cancer during chemotherapy with a combination of S-1 and cisplatin. The regimen of S-1 and cisplatin is widely used for treatment of human epidermal growth factor receptor 2 (HER2)-negative gastric cancer as first-line therapy recommended by the Japanese Gastric Cancer Association. To the best of our knowledge, this is the first reported case of TMA in association with the use of S1 and cisplatin.

\section{Case Report}

A 77-year-old Japanese woman was diagnosed with advanced gastric cancer with multiple lymph node and liver metastases. 

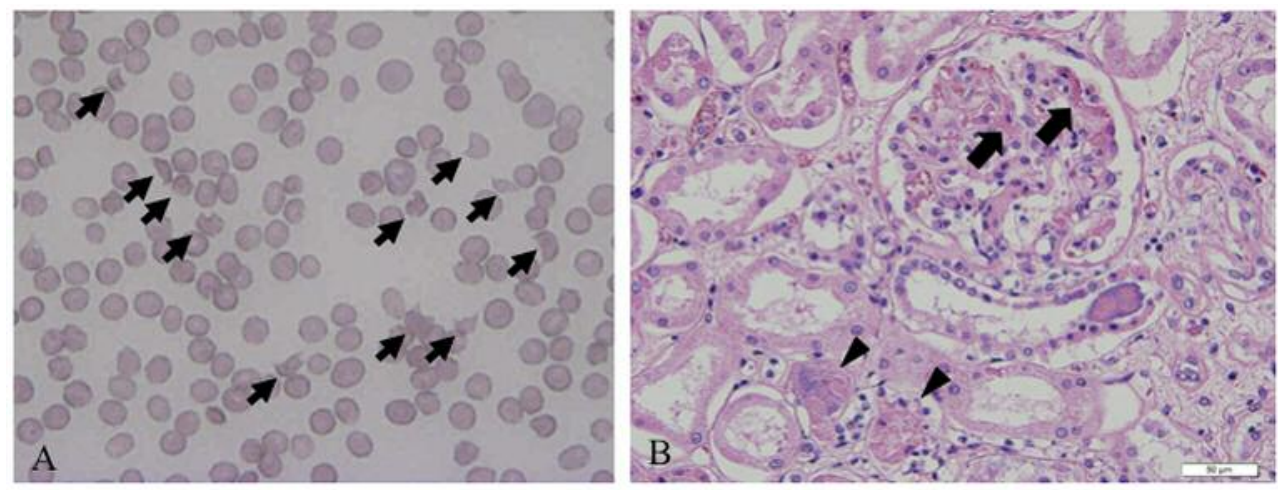

Figure 1. Pathological findings. (A) Schistocytes (arrow) in peripheral blood smear. (B) Microthrombus in the glomeruli (arrow) and tubulonecrosis (arrowhead).

Because her HER2 score was 0 , she received chemotherapy with a combination of $\mathrm{S}-1$ and cisplatin. She was administered S-1 $\left(80 \mathrm{mg} / \mathrm{m}^{2}\right)$ from day 1 to 21 and cisplatin $\left(60 \mathrm{mg} / \mathrm{m}^{2}\right)$ on day 8 , while no drugs were given from day 22 to 35. Although no significant adverse events occurred during the first or second course of chemotherapy, she was admitted to the hospital with anemia and thrombocytopenia with a normal white blood cell count on day 17 of the third course. All medications, including S-1, were discontinued. She received a transfusion of red blood cells and thrombocytes; however, neither anemia nor thrombocytopenia improved. She was diagnosed with hemolytic anemia, with schistocytes being observed in a peripheral blood smear (Figure 1A). Her blood urea nitrogen concentration gradually increased and severe proteinuria was observed. Her body temperature was within normal limits, her consciousness was clear and no abnormal neurological findings were observed until the last day. The plasma activity of von Willebrand factor was $>200 \%$, the activity of ADAMTS13 was $37.9 \%$, while she was anti-ADAMTS13 autoantibody-negative. Although the examination results did not meet the diagnostic criteria for TTP, we diagnosed the patient with TMA based on her clinical state of thrombocytopenia, schistocytosis, hemolytic anemia and renal dysfunction. She underwent treatment with methylprednisolone (1,000 mg/day for 3 days), recombinant human soluble thrombomodulin and plasmapheresis. However, her response to treatment was poor; she lost consciousness on day 15 of hospitalization and died on day 16.

An autopsy was performed. The gastric cancer had invaded the serosa and a broad area of the proper muscular layer of the stomach, whereas a limited area of the mucosal membrane was involved. Metastatic cells were observed in the lymph nodes, liver, bilateral adrenal glands, thymus, lower esophagus and spinal marrow. Microthrombi were observed in the glomeruli and tubulonecrosis was observed in the kidneys (Figure 1B). However, no microthrombi were found in the brain or other organs.

\section{Discussion}

Thrombocytopenia with multiple organ failure is a risk factor for mortality $(1,2)$. In the present case, we first thought that the patient's thrombocytopenia and anemia were due to myelosuppression from anticancer chemotherapy. However, the patient's response to transfusion was poor, while schistocytosis was found microscopically. Symptoms of thrombocytopenia, hemolytic anemia and renal failure were present, but fever and neurological failure were absent. The activity of ADAMTS13 was $37.9 \%$ and anti-ADAMTS 13 autoantibody was negative. The case did not meet the diagnostic criteria for TTP; thus, TMA was diagnosed.

The management of drug-induced TMA is poorly understood, although plasmapheresis is considered the standard treatment for idiopathic TTP (1). Bendapudi et al. have reported that the mortality rate associated with an ADAMTS13 activity of $>10 \%$ is higher than that associated with an ADAMTS13 activity of $\leq 10 \%$ (3).

TMA is a syndrome with multiple etiologies defined, however, by microangiopathic hemolytic anemia, thrombocytopenia and microvascular thrombosis. Cancerrelated TMA, such as that associated with lymphoma, breast cancer, lung cancer and gastric cancer, has been reported (9, 10). Bone marrow metastasis is also a reported risk factor for TMA (9). Therefore, in the present case, TMA might have developed even if the patient had not been treated with S-1 and cisplatin. Many TMA patients reportedly have a drug-induced etiology, including anticancer drugs, such as gemcitabine, mitomycin, docetaxel, oxaliplatin, vincristine and anti-vascular endothelial growth factor agents (4). The association between TMA and anticancer drugs is difficult to elucidate because malignancy itself may cause TMA $(5,6)$. 
The regimen of S-1 and cisplatin is widely used for treatment of HER2-negative gastric cancer as first-line therapy recommended by the Japanese Gastric Cancer Association. Additionally, no cases of TMA in association with the use of S-1 and cisplatin have been previously reported. This report may help determine appropriate therapy for future cases.

\section{Conflicts of Interest}

The Authors declare no conflicts of interest exist with regards to the present study.

\section{References}

1 Zheng XL: ADAMTS13 and von Willebrand factor in thrombotic thrombocytopenic purpura. Ann Rev Med 66: 211$225,2015$.

2 Nishijima Y, Hirata H, Himeno A, Kida H, Matsumoto M, Takahashi R, Otani Y, Inoue K, Nagatomo I, Takeda Y, Kijima T, Tachibana I, Fujimura Y and Kumanogoh A: Drug-induced thrombotic thrombocytopenic purpura successfully treated with recombinant human soluble thrombomodulin. Internal Med 52: 1111-1114, 2013.

3 Bendapudi PK, Li A, Hamdan A, Uhl L, Kaufman R, Stowell C, Dzik W and Makar RS: Impact of severe ADAMTS13 deficiency on clinical presentation and outcomes in patients with thrombotic microangiopathies: The experience of the Harvard TMA Research Collaborative. Br J Haemat 171: 836-844, 2015.
4 Al-Nouri ZL, Reese JA, Terrell DR, Vesely SK and George JN: Drug-induced thrombotic microangiopathy: a systematic review of published reports. Blood 125: 616-618, 2015.

5 Lesesne JB, Rothschild N, Erickson B, Korec S, Sisk R, Keller J, Arbus M, Woolley PV, Chiazze L, Schein PS and Neefe JR: Cancer-associated hemolytic-uremic syndrome: analysis of 85 cases from a national registry. J Clin Oncol 7: 781-789, 1989.

6 Izzedine $\mathrm{H}$ and Perazella MA: Thrombotic microangiopathy, cancer, and cancer drugs. Am J Kidney Dis 66: 857-868, 2015.

7 Furlan M, Robles R, Galbusera M, Remuzzi G, Kyrle PA, Brenner B, Krause M, Scharrer I, Aumann V, Mittler U, Solenthaler M and Lammle B: von Willebrand factor-cleaving protease in thrombotic thrombocytopenic purpura and the hemolytic-uremic syndrome. New Engl J Med 339: 1578-1584, 1998.

8 Tsai HM and Lian EC: Antibodies to von Willebrand factorcleaving protease in acute thrombotic thrombocytopenic purpura. New Engl J Med 339: 1585-1594, 1998.

9 Govind Babu K and Bhat GR: Cancer-associated thrombotic microangiopathy. Ecancermedicalscience 10: 649, 2016.

10 Lechner K and Obermeier HL: Cancer-related microangiopathic hemolytic anemia: Clinical and laboratory features in 168 reported cases. Medicine 91: 195-205, 2012.
Received January 23, 2017

Revised March 3, 2017

Accepted March 6, 2017 